8. Antonescu CR, Dickson BC, Sung YS, et al. Recurrent YAP1 and MAML2 rearrangements in retiform and composite hemangioendothelioma. Am J Surg Pathol 2020; 44: 1677-84.

DOI: https://doi.org/10.1016/j.pathol.2021.02.009

\section{Intra-abdominal Pacinian corpuscle mimicking a peritoneal tumour deposit}

Sir,

Pacinian corpuscles are most commonly encountered in the skin but may be found at other locations, in particular in the peripancreatic mesentery, ${ }^{1}$ where their occurrence may be unexpected and lead to diagnostic confusion. We present a case of peritoneal Pacinian corpuscle identified at laparotomy, which was thought clinically to represent a metastatic tumour deposit.

A 71-year-old woman with no significant past medical history presented with biliary colic and underwent laparoscopic cholecystectomy. Pathologic examination of the gallbladder revealed an unexpected adenocarcinoma [American Joint Committee on Cancer (AJCC) stage pT1b] ${ }^{2}$ arising from intestinal type adenoma. Subsequent staging investigations revealed no evidence of distant metastases and the patient subsequently came forward for segment IVb/V liver resection and portal lymphadenectomy to ensure surgical clearance of the primary. At laparotomy a peritoneal nodule suspicious for metastasis was discovered, and submitted for frozen section examination. Sections from the peritoneal biopsy revealed a Pacinian corpuscle in close association with a nerve (Fig. 1).

Pacinian corpuscles are pressure receptors, hundreds of which are usually located in the dermis of the hand. The corpuscle transmits pressure stimuli via numerous lamellae of connective tissue separated by fluid, to a central partially myelinated nerve fibre. ${ }^{3}$ Anomalous Pacinian corpuscles may include a solitary hypertrophied corpuscle to double or multiple structures, some in varying stages of development. A neuroma can arise from a Pacinian corpuscle; these are usually found in the hand and typically present with pain.

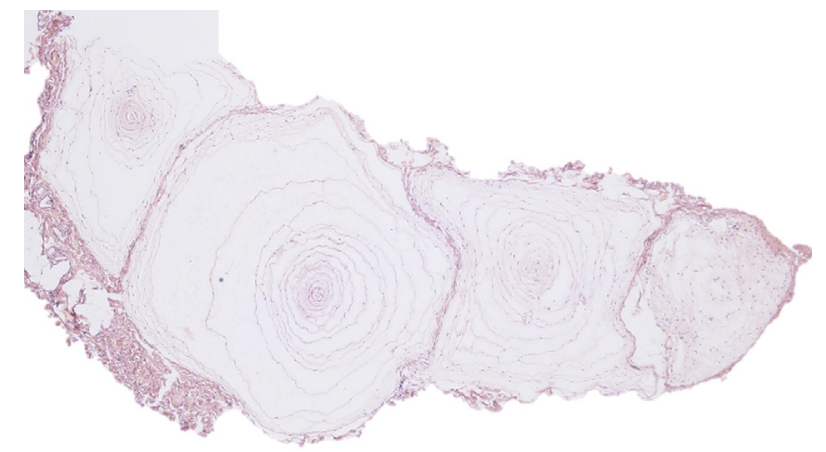

Fig. 1 The tissue submitted for frozen section revealed typical appearances of a Pacinian corpuscle $(\mathrm{H} \& \mathrm{E})$
They have also rarely been reported in the vulva, foot and sacrococcygeal area. ${ }^{3}$ A prior history of trauma or recurrent pressure in the relevant anatomical location has been reported by several authors ${ }^{3,4,6}$ and it is postulated that such trauma to nerves or normal Pacinian corpuscles may be a risk factor for development of a Pacinian corpuscle neuroma. ${ }^{6}$

Dembinski and Jones ${ }^{4}$ describe an intra-abdominal Pacinian neuroma seen macroscopically as white lesions on the adventitia of the aorta, clinically considered suspicious for metastatic colonic adenocarcinoma. This patient had an infrarenal aortic aneurysm and the area may have been subjected to pulsatile pressure/trauma. Stouder and McDonald report a case in which multiple (more than 30) pale nodules were present on the serosa overlying the site of a previous gastrojejunostomy (for duodenal ulcer), and in the adjacent omentum. It is reported that these nodules macroscopically resembled tumour implants on the serosa, but histological examination confirmed enlarged Pacinian corpuscles. More recently, Standop et $a l^{7}{ }^{7}$ reported histologically confirmed Pacinian corpuscles within the pancreas of a 76-year-old female with cancer in the head of the pancreas, and we have similar cases in our files. The purpose of intra- and peripancreatic Pacinian corpuscles is undetermined but may be involved in the regulation of blood flow, detection of changes in the vibration, pressure and/or distension of the peritoneum or pancreas and possibly in the perception of abdominal pain. ${ }^{8}$

In summary, Pacinian corpuscles may be found at unusual anatomical locations and may be confused clinically with tumour deposits; however, thanks to their characteristic appearance, frozen section examination can elucidate the true nature of such lesions, allowing planned surgery to proceed. In particular, pathologists and surgeons should be aware of their frequent occurrence in peripancreatic tissues.

Conflicts of interest and sources of funding: The authors state that there are no conflicts of interest to disclose.

\section{Anna Eccles ${ }^{1}$, Chris Hemmings ${ }^{1,2}$}

${ }^{1}$ Canterbury Health Laboratories, Christchurch, New Zealand; ${ }^{2}$ Christchurch Clinical School, University of Otago School of Medicine, Christchurch, New Zealand

Contact A/Prof Chris Hemmings.

E-mail: chris.hemmings@cdhb.health.nz

1. Stouder DJ, McDonald LW. Enlarged intra-abdominal Pacinian corpuscles simulating tumour implants. Am J Clin Pathol 1968; 49: 79-83.

2. Amin M, editor. AJCC Cancer Staging Manual. 8th ed. New York: Springer, 2017; 303-9.

3. Shah P, Kareliya H, Salome, Toprani T. Pacinian corpuscle tumour. Int J Med Health Res 2019; 5: 48-51.

4. Dembinski AS, Jones JW. Intra-abdominal Pacinian neuroma: a rare lesion in an unusual location. Histopathology 1991; 19: 89-90.

5. Narayanamurthy VB, Winston AT, Gupta A. A rare case of Pacinian corpuscle neuroma. Can J Plast Surg 2005; 13: 43-5.

6. Zanardi F, Cooke RMT, Maiorana A, et al. Is this case of a very rare disease work-related? A review of reported cases of Pacinian neuroma. Scand J Work Environ Health 2011; 37: 253-8.

7. Standop J, Ulrich A, Schneider MB, Andren-Sandberg A, Pour PM. Pacinian corpuscle in the human pancreas. Pancreas 2001; 23: 36-9.

8. Garcia-Suarez O, Calavia MG, Perez-Molto FJ, et al. Immunohistochemical profile of human pancreatic pacinian corpuscles. Pancreas 2010; 39: 403-10. 